\title{
The velocity potential and the interacting force for two spheres moving perpendicularly to the line joining their centers
}

\author{
L. LI, W.W. SCHULTZ and H. MERTE, Jr. \\ Department of Mechanical Engineering, University of Michigan, Ann Arbor, Michigan 48109-2125, USA
}

Received 25 September 1991; accepted in revised form 20 July 1992

\begin{abstract}
The velocity potential around two spheres moving perpendicularly to the line joining their centers is given by a series of spherical harmonics. The appropriateness of the truncation is evaluated by determining the residual normal surface velocity on the spheres. In evaluating the residual normal velocity, a recursive procedure is constructed to evaluate the spherical harmonics to reduce computational effort and truncation error as compared to direct transformation or numerical integration. We estimate the lift force coefficient for touching spheres to be 0.577771 , compared to the most accurate earlier estimate of 0.51435 by Miloh (1977).
\end{abstract}

\section{Introduction}

Vapor bubbles generated on a flat heating surface in forced convection boiling can be subjected to a bulk flow parallel to the heating surface and to the lift produced due to the pressure distribution. This lift may be of particular significance for those applications in which forced convection boiling is taking place at the microgravity conditions of space. Limiting cases for the general problem of a sphere at the vicinity of a wall are when the Reynolds number is very high and very low.

Leighton and Acrivos [1] investigated the lift on a small sphere touching a plane with a simple shear flow and concluded that the lift is too small to be significant relative to the drag at Reynolds numbers of $O\left(10^{-2}\right)$. Drew [2], investigating the lift force with Poiseuille flow in a channel for small Reynolds number $(\operatorname{Re} \ll 1$ ), pointed out that the slip-shear interaction is decreased when the sphere is sufficiently close to the wall and enhanced when it is further away, and the magnitude of the wall contribution is too small to change the trajectory of the sphere. His result also showed that the lift force changes sign when the sphere is approximately three diameters from the wall. Cherukat and McLaughlin [3] experimentally examined the migration velocity of rigid spheres sedimenting near a large flat vertical wall with Reynolds number based on the diameter of the sphere in the range of $0.1-10$. Their results of the migration velocity and the dependence of the migration velocity on the distance from the wall matches fairly well with the predictions of Vasseur and Cox [4] for Reynolds number up to 3.0. They also observed that a sphere touching the wall does not migrate as it sediments.

Here we shall examine the limiting case of inviscid flow, which in some sense represents the high Reynolds number flow around vapor bubbles. It is well known that high Reynolds number flow around a vapor bubble is effectively inviscid, since the shear stress at the interface is negligible compared with that for a solid sphere due to internal circulation. Therefore the existing solutions for the lift force for the cases of solid spheres at low Reynolds number flow may not apply to the case considered here. This reduces the problem to a potential flow of two spheres moving perpendicularly to the line joining their centers. 
The problem was first considered by Hicks [5], among others. Their solutions used the method of images to determine the successive locations and strengths of dipoles that are added to the potential of a single moving sphere. Their method is not very effective and only approximate solutions were attained. Basset [6] gave an approximate solution by another kind of imaging method: the added terms are found by the transformation of spherical harmonics rather than by locating dipoles. Basset, as well as others, may not have realized that the procedure could also lead to an exact solution if terms of higher-degree, first-order spherical harmonics are retained. Herman [7], with a method similar to that of Basset, gave the potential as a doubly infinite sum of derivative operators applied to the first order tesseral harmonics with increasing degree. Due to the complexity of the expression, he stated that it was incapable of full interpretation. Endo [8] gave the velocity potential in terms of bipolar coordinates, but was unable to provide an explicit expression for the coefficients of the potential. The bipolar coordinates system was also used by Love [9] to give an exact potential for the two-sphere problem. Weihs and Small [10] show that the bipolar coordinate system should not be used when two spheres are touching. Voinov [11] gave the potential as an infinite sum of repeated integrals in symbolic form by determining the location and strength of the dipoles. However, the exact expression of each term was not clear. Miloh [12] solved for the potential function as a series of spherical harmonics. The coefficients were determined by solving a truncation of an infinite set of equations by applying the boundary conditions. Here, we used the successive imaging method to give a general explicit expression for the coefficients. Since the velocity potential is given as an infinite series, the question of adequate truncation must be addressed. This is especially important when the two spheres are very close or touching, since the rate of convergence decreases. This is studied for the first time in the present work by examining the residual normal velocity on the sphere surface for different truncations of the potential function.

In section II, the successive imaging method is used to construct the velocity potential for the case of two touching spheres with different truncations and for two spheres with various separations. In section III, the residual normal velocity is evaluated for two touching spheres with different truncations of the velocity potential. To avoid truncation errors or lengthy numerical computation in computing the residual normal velocity, a recursive procedure is constructed to evaluate spherical harmonics on a spherical surface which does not have the same origin as the harmonics. In section IV, the interacting force on a sphere is found by directly integrating the local pressure on the spherical surface.

\section{The construction of the velocity potential}

The case of two spheres of radii $R_{1}$ and $R_{2}$ moving in an ideal liquid at rest at infinity, with steady velocities $U_{1}$ and $U_{2}$, respectively, in the negative $X$ direction is shown in Fig. 1 . Spherical coordinates $r_{1}, \theta_{1}, \varphi$, and $r_{2}, \theta_{2}, \varphi$ are fixed at the centers of the spheres at $O_{1}$ and $\mathrm{O}_{2}$, respectively. The Cartesian coordinate system has its origin at the mid-point on the line joining the centers of the two spheres with the $X$-axis perpendicular to this line. The polar angles $\theta_{1}$ and $\theta_{2}$ are measured from the $Z$-axis, and the angle $\varphi$ is measured from $X$-axis in the $X-Y$ plane. Although the general case will be retained for now, the particular case of interest is where $U_{1}=U_{2}, R_{1}=R_{2}$, for which case the surface $z=0$ acts as a wall.

The total velocity potential $\Phi$ at any point outside the spheres is the sum of that produced by the sphere of radius $R_{1}$ and by the sphere of radius $R_{2}$, i.e. 


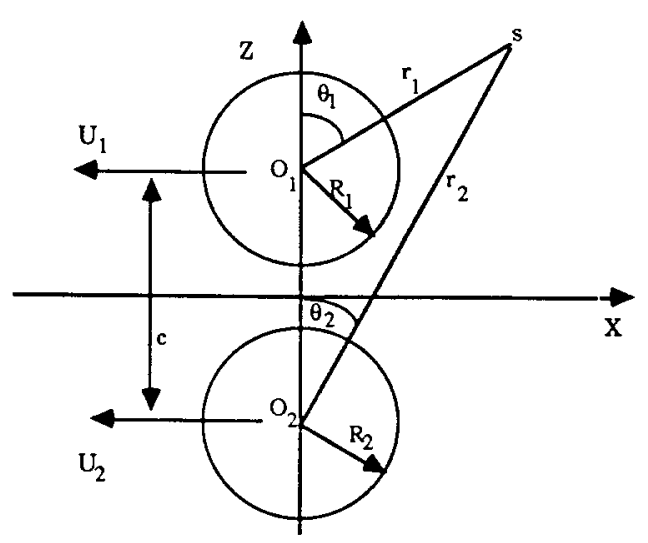

Fig. 1. Two spheres moving perpendicularly to the line joining their centers.

$$
\Phi=\cos \varphi \sum_{m=1}^{2} \Phi_{m}
$$

and the boundary conditions on the sphere surfaces are

$$
\begin{aligned}
& \left.\frac{\partial \Phi_{m}}{\partial r_{m}}\right|_{r_{m}=R_{m}}=-U_{m} p_{1 m}^{1}, \\
& \left.\frac{\partial \Phi_{m}}{\partial r_{3-m}}\right|_{r_{3-m}=R_{3-m}}=0, m=1,2,
\end{aligned}
$$

where $p_{n m}^{1}$ is the $n$ th-degree associated Legendre polynomial of the first-order (surface spherical harmonic) with center at $O_{m}$.

The velocity potentials that satisfy the boundary conditions are found by the successive imaging method. The essence of this method relies on the following relations between the two solid spherical harmonics $p_{n m}^{1} / r_{m}^{n+1}(m=1,2)$ given by Basset [6]:

$$
\begin{aligned}
& \frac{p_{n 2}^{1}}{r_{2}^{n+1}}=\frac{r_{1}}{(n-1) ! c^{n+2}} \sum_{k=0}^{\infty}(-1)^{k} \frac{(n+1+k) !}{(2+k) !}\left(\frac{r_{1}}{c}\right)^{k} p_{1+k, 1}^{1},\left(r_{1}<c\right), \\
& \frac{(-1)^{n-1} p_{n 1}^{1}}{r_{1}^{n+1}}=\frac{r_{2}}{(n-1) ! c^{n+2}} \sum_{k=0}^{\infty} \frac{(n+1+k) !}{(2+k) !}\left(\frac{r_{2}}{c}\right)^{k} p_{1+k, 2}^{1},\left(r_{2}<c\right) .
\end{aligned}
$$

The successive imaging method is now briefly described. The individual potentials in (1) for each $m$ is first approximated by the potential of a single sphere moving in an unbounded liquid that satisfies the nonhomogeneous but not the homogeneous condition in (2) for corresponding $m$. Relation (3) (if $m=2$ ) or (4) (if $m=1$ ) is then used to express this potential in terms of the other coordinates, and appropriate spherical harmonics are added to satisfy the homogeneous condition in (2). The added harmonics are then expressed in the original coordinates using (4) (if $m=2$ ) or (3) (if $m=1$ ), and other spherical harmonics must be added to satisfy the originally nonhomogeneous boundary condition in (2). This procedure is repeated with successive terms to satisfy the boundary conditions alternately. Details of the above are given in the appendix. The final results are given below: 


$$
\begin{aligned}
\Phi_{m}= & U_{m} \frac{R_{m}^{3}}{2 r_{m}^{2}} p_{1 m}^{1}+U_{m} \frac{D_{m}^{3}}{2}\left\{\sum_{i=1}^{\infty} \frac{i}{i+1} D_{3-m}^{i-1} R_{3-m}^{i+2} \frac{p_{i, 3-m}^{1}}{r_{3-m}^{i+1}}\right. \\
& \left.+\sum_{j=1}^{\infty} \sum_{i=1}^{\infty}(-1)^{j-1} \frac{j}{(j+1)(j+1) !} D_{m}^{j-1} R_{m}^{j+2} \frac{p_{j m}^{1}}{r_{m}^{j+1}} \frac{i^{2}(i+j) !}{(i+1) !} D_{(3-m)}^{2 i+1}+\ldots\right\}, m=1,2 .
\end{aligned}
$$

For terms in (5) with summation orders higher than 2, a generalized expression can be given as

$$
\begin{gathered}
\sum_{i_{k}=1}^{\infty} \cdot \ldots \cdot \sum_{i_{2}=1}^{\infty} \sum_{i_{1}=1}^{\infty}(-1)^{(k-1)\left(i_{k}-1\right)} \frac{i_{k} D_{M}^{i_{k}-1} R_{M}^{i_{k}+2}}{\left(i_{k}+1\right)\left(i_{k}+1\right) !} \frac{p_{i_{k} M}^{1}}{r_{M}^{\left(i_{k}+1\right)}} \cdot \ldots \\
\cdot \frac{i_{2}^{2}\left(i_{2}+i_{3}\right) !}{\left(\left(i_{2}+1\right) !\right)^{2}} D_{m}^{2 i_{2}+1} \frac{i_{1}^{2}\left(i_{1}+i_{2}\right) !}{\left(i_{1}+1\right) !} D_{3-m}^{2 i_{1}+1}
\end{gathered}
$$

where $M=(3-m) \delta\left((-1)^{k+1}-1\right)+m \delta\left((-1)^{k}-1\right), D_{m}=R_{m} / c, \delta(x)$ is the Kronecker delta function, which is one for $x=0$ and zero otherwise. Substituting (5) into (1) and collecting terms with the same $p_{n m}^{1}$ results in

$$
\Phi=U_{1} \phi^{\prime} \cos \varphi
$$

where $\phi^{\prime}$ is the amplitude for the potential of unit velocity in the direction of $U_{1}$, given by

$$
\phi^{\prime}=\frac{R_{1}^{3}}{2 r_{1}^{2}} p_{1,1}^{1}+\frac{U_{2}}{U_{1}} \frac{R_{2}^{3}}{2 r_{2}^{2}} p_{1,2}^{1}+\lim _{N \rightarrow \infty} \sum_{n=1}^{N} \sum_{m=1}^{2}(-1)^{(n-1) \delta(m-1)} A_{n m} R_{m}^{n+2} \frac{1}{r_{m}^{n+1}} p_{n m}^{1}, m=1,2
$$

where $A_{n m}$ are the coefficients and are given by the following infinite series:

$$
\begin{aligned}
A_{n m}= & \frac{n}{n+1} D_{m}^{n-1}\left\{\left(\frac{U_{2}}{U_{1}}\right)^{\delta(m-1)} D_{3-m}^{3}+\left(\frac{U_{2}}{U_{1}}\right)^{\delta(m-2)} \frac{D_{m}^{3}}{2} \sum_{i=1}^{\infty} \frac{i^{2}(i+n) !}{(n+1) !(i+1) !} D_{3-m}^{2 i+1}\right. \\
& +\left(\frac{U_{2}}{U_{1}}\right)^{\delta(m-1)} \frac{D_{3-m}^{3}}{2} \sum_{j=1}^{\infty} \sum_{i=1}^{\infty} \frac{j^{2}(j+n) !}{(n+1) !(j+1) !} D_{3-m}^{2 j+1} \cdot \\
& \left.\cdot \frac{i^{2}(i+j) !}{(j+1) !(i+1) !} D_{m}^{2 i+1}+\ldots\right\}, m=1,2 .
\end{aligned}
$$

A generalized expression for terms in (8) with summation orders higher than 2 can be given as

$$
\begin{aligned}
& \left(\frac{U_{2}}{U_{1}}\right)^{\delta(m-L)} \frac{D_{m}^{3}}{2} \sum_{i_{k}=1}^{\infty} \sum_{i_{k-1}=1}^{\infty} \ldots \cdot \sum_{i_{1}=1}^{\infty} \frac{i_{k}^{2}\left(i_{k}+n\right) !}{(n+1) !\left(i_{k}+1\right) !} D_{3-m}^{2 i_{k}+1} \frac{i_{k-1}^{2}\left(i_{k-1}+i_{k}\right) !}{\left(i_{k}+1\right) !\left(i_{k-1}+1\right) !} \cdot D_{m}^{2 i_{k-1}+1} \\
& \quad \ldots \cdot \frac{i_{1}^{2}\left(i_{1}+i_{2}\right) !}{\left(i_{2}+1\right) !\left(i_{1}+1\right) !} D_{M}^{2 i_{1}+1}
\end{aligned}
$$

where $L=1+\left(1-(-1)^{k}\right) / 2$ and the subscript $M$ is the same as in (5a). A minor point to be made here is that it can be shown that for $D<1$, which is the case in this work, a closed 
Table 1. The coefficients $A_{n}$ vs. N for touching spheres

\begin{tabular}{rlllll}
\hline$n \backslash N$ & 30 & 100 & 500 & 1000 & 2000 \\
\hline 1 & 0.04032383 & 0.04034059 & 0.04034132 & 0.04034133 & 0.04034133 \\
5 & 0.00830055 & 0.00834003 & 0.00834175 & 0.00834177 & 0.00834177 \\
10 & 0.00176079 & 0.00181483 & 0.00181718 & 0.00181720 & 0.00181721 \\
30 & 0.00005986 & 0.00013832 & 0.00014210 & 0.00014214 & 0.00014214 \\
100 & & 0.00000205 & 0.00000800 & 0.00000807 & 0.00000808 \\
\hline
\end{tabular}

Table 2. The coefficients $A_{n}$ vs. $c / R$

\begin{tabular}{rlllllll}
\hline$n \backslash \frac{c}{R}$ & 2.000000 & 2.000001 & 2.000010 & 2.000100 & 2.001000 & 2.010000 & 2.100000 \\
\hline 1 & 0.04034133 & 0.04341190 & 0.04339905 & 0.04032728 & 0.04020703 & 0.03915365 & 0.03195988 \\
5 & 0.00834177 & 0.00834162 & 0.00834030 & 0.00832765 & 0.00821460 & 0.00738535 & 0.00388762 \\
10 & 0.00181721 & 0.00181709 & 0.00181606 & 0.00180643 & 0.00172752 & 0.00127349 & 0.00027221 \\
50 & 0.00004233 & 0.00004227 & 0.00004178 & 0.00003806 & 0.00002138 & 0.00000149 & 0.00000000 \\
100 & 0.00000808 & 0.00000803 & 0.00000771 & 0.00000578 & 0.00000131 & 0.00000000 & 0.00000000 \\
\hline
\end{tabular}

form solution exists for the first summation term in (8) as

$$
\begin{aligned}
\sum_{i=1}^{\infty} \frac{i^{2}(i+n) !}{(n+1) !(i+1) !} D_{m}^{2 i+1}= & \frac{1}{n(n+1)}\left\{\frac{1}{D_{m}}\left(\frac{1}{\left(1-D_{m}^{2}\right)^{n}}-1\right)-\frac{n D_{m}}{\left(1-D_{m}^{2}\right)^{n+1}}\right. \\
& \left.+\frac{n(n+1) D_{m}^{3}}{\left(1-D_{m}^{2}\right)^{n+2}}\right\} .
\end{aligned}
$$

We note that (8) can be transformed to an infinite set of equations, as given by Miloh. Also, when $R_{1}=R_{2}$ and $U_{1}=U_{2}$, then $A_{n 1}=A_{n 2}=A_{n}$, and only one set need be computed for each separation. Table 1 gives selected values of the coefficients $A_{n}$ in the velocity potential for the case of two identical touching spheres, with various truncation $N$.

When the distance between the two identical spheres is sufficiently large $(c / R \geqslant 2.02)$, the computation of the coefficients using (8) converges very rapidly. Selected values of the coefficients $A_{n}$ for various separations are given in Table 2, where $N$ varies from 50 for $c / R=2.2$ to 2000 for $c / R=2$ (touching spheres).

\section{Residual normal velocity on the sphere surface}

The adequacy of the velocity potential constructed above can be evaluated using the residual normal velocity on either spherical surface. Since the residual normal velocity per unit stream velocity at any given $\theta$ is given by $v_{n r}^{\prime}=\left.v_{n r}^{\prime}\right|_{\varphi=0} \cos \varphi$, its evaluation at $\varphi=0$ alone is sufficient, and $v_{n r}^{\prime}$ is used henceforth to denote the residual normal velocity at $\varphi=0$. It is computed by differentiating (7) with respect to $r_{1}$ and setting $r_{1}=R_{1}$, i.e.:

$$
\begin{aligned}
\left.v_{n r}^{\prime}\right|_{r_{1}=R_{1}}= & -p_{1,1}^{1}+\left.\frac{U_{2}}{U_{1}} \frac{\partial}{\partial r_{1}}\left(\frac{R_{2}^{3}}{2 r_{2}^{2}} p_{1,2}^{1}\right)\right|_{r_{1}=R_{1}}+\lim _{N \rightarrow \infty} \sum_{n=1}^{N}(n+1) \\
& \times\left\{(-1)^{n} A_{n} p_{n 1}^{1}+\left.B_{n} \frac{\partial}{\partial r_{1}}\left(\frac{R_{2}^{n+2}}{n+1} \frac{1}{r_{2}^{n+1}} p_{n 2}^{1}\right)\right|_{r_{1}=R_{1}}\right\} .
\end{aligned}
$$


The recursive scheme given by Hobson [13],

$$
(n-m) p_{n}^{m}(\mu)=(2 n-1) \mu p_{n-1}^{m}(\mu)-(n-1+m) p_{n-2}^{m}(\mu),
$$

together with the initial values $p_{11}^{1}=\sin \theta_{1}$ and $p_{01}^{1}=0$ are used to evaluate $p_{n 1}^{1}$ in (9).

The evaluation of the second term inside the summation sign on the right-hand side of (9) is somewhat difficult. One possibility is to express this in terms of the coordinates $r_{1}$ and $\mu_{1}$ by using (3). However, the truncation of an infinite series arises, with an unknown truncation error. To avoid the truncation error the term referred to above can be evaluated by using the integral definition of the associated Legendre polynomial:

$$
\left.\frac{\partial}{\partial r_{1}}\left(\frac{R_{2}^{n+2}}{n+1} \frac{1}{r_{2}^{n+1}} p_{n 2}^{1}\right)\right|_{r_{1}=R_{1}}=\frac{R_{2}^{n+2} \sin \theta_{1}}{R_{1}^{n+2}}\left\{\frac{\left(D_{1}^{-2}-1\right) n-D_{1}^{-1} \mu_{1}-1}{n+1} K_{n}-D_{1}^{-1} K_{n-1}\right\},
$$

where

$$
K_{n}=\frac{n(n+1)}{\pi\left(1+D_{1}^{-2}+2 D_{1}^{-1} \mu_{1}\right)^{n+3 / 2}} \int_{0}^{\pi}\left(\mu_{1}+D_{1}^{-1}+\sqrt{\mu_{1}^{2}-1} \cos \alpha\right)^{n-1} \sin ^{2} \alpha \mathrm{d} \alpha .
$$

Since a simple closed form solution for the integral does not exist, and numerical integration would be time consuming and error prone, a recursive procedure is constructed instead:

$$
K_{n}=\left[(n-1)\left(1+D_{1}^{-2}+2 D_{1}^{-1} \mu_{1}\right)\right]^{-1}\left\{(2 n-1)\left(D_{1}^{-1}+\mu_{1}\right) K_{n-1}-n K_{n-2}\right\}
$$

with the initial values of

$$
\begin{aligned}
& K_{1}=\left(1+D_{1}^{-2}+2 D_{1}^{-1} \mu_{1}\right)^{-5 / 2}, \\
& K_{0}=0 .
\end{aligned}
$$

The residual normal velocity on the sphere surface at $\varphi=0$ and between the polar angle $\theta=140$ to 180 is plotted in Fig. 2 for two identical touching spheres for the cases where 30 and 1000 terms in the velocity potential are evaluated and used. The same is plotted in Fig. 3 for the cases where 2000 coefficients in the velocity potential are evaluated, but only the first 30 and 1000 terms are used.

From Figs. 2 and 3, it can be seen that as the number of terms in the velocity potential increases, the amplitude of the residual normal velocity at the sphere surface diminishes, and the region having a higher amplitude narrows.

The root mean square of the residual normal velocity $E$ on the sphere at $\varphi=0$ and for $\theta$ between 0 and $\pi$ is defined by

$$
E^{2}=\frac{1}{\pi} \int_{0}^{\pi} v_{n}^{\prime 2} \mathrm{~d} \theta
$$

These are plotted in Fig. 4 for the two cases where the potentials are summed to $N$ terms, 


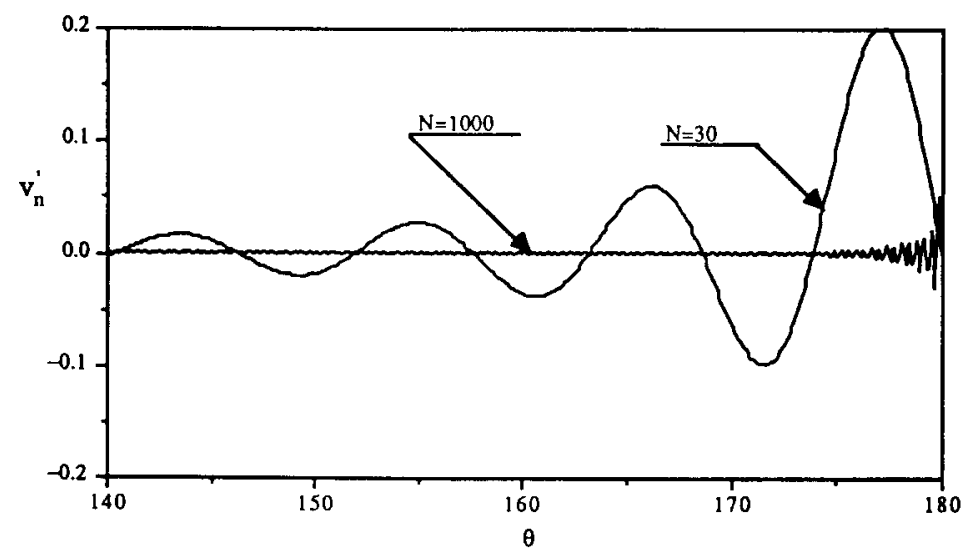

Fig. 2. Residual normal velocity for two touching spheres with unit velocity $V_{n}^{\prime}$ vs. $\theta$ (the polar angle), at $\varphi=0$, for the velocity potential evaluated with 30 and 1000 terms.

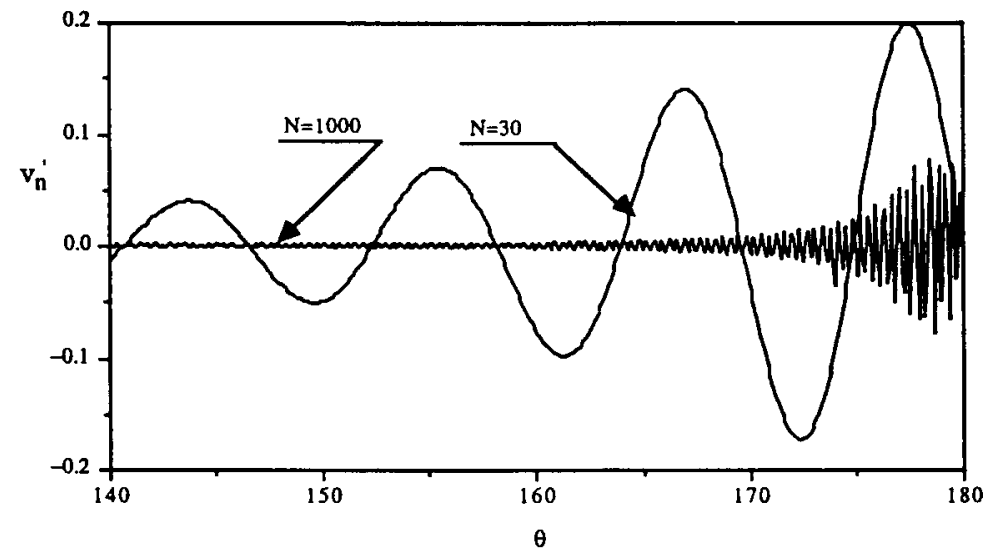

Fig. 3. Residual normal velocity for two touching spheres with unit velocity $V_{n}^{\prime}$ vs. $\theta$ (the polar angle), at $\varphi=0$, for the velocity potential that uses the first 30 and 1000 terms of the coefficients computed to 2000 terms.

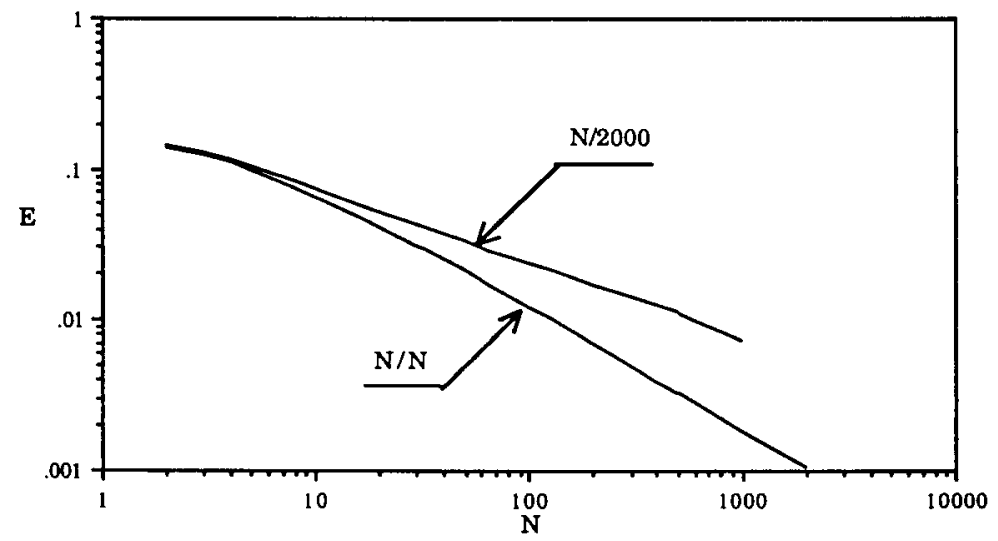

Fig. 4. The root mean square of the normal residual velocity $E$ vs. $N$-the number of terms in the potential used. 
but the coefficients are computed with $N$ and 2000 terms, indicated by $N / N$ and $N / 2000$, respectively.

Figures 2, 3 and 4 show that the residual normal velocities of a potential using the first $N$ terms of the potential computed with 2000 terms are considerably larger than those that are computed with $N$ coefficients. Therefore, if a velocity potential with fewer terms is desired, the coefficients should be computed specifically for that number rather than simply truncating an expression that has more terms. Figure 4 shows that the convergence rate of $E$ for $N / N$ is approximately $N^{-.8}$ while that for $N / 2000$ is $N^{-.5}$.

\section{The interacting force on a sphere}

To determine the interacting force on the spheres, the unsteady velocity potential is transformed into a steady one:

$$
\Phi_{S}=U_{1} r_{1} \sin \theta_{1} \cos \varphi+\Phi
$$

If body force is neglected, the pressure variation $\delta p$ at any point in the fluid is given by the well-known Bernoulli's theorem as

$$
\delta p=p-p_{0}=-\frac{1}{2} \rho_{1}\left(v_{r}^{2}+v_{\theta}^{2}+v_{\varphi}^{2}\right)
$$

The net interacting force between the spheres can be found by integrating $\delta p$ over either of the sphere surfaces. Here it will be done on the sphere at $O_{1}$. If the error introduced by truncating Eq. (7) to a finite number of terms is neglected, then on the surface of the sphere at $O_{1}, v_{r}=0$ and

$$
\begin{aligned}
& v_{\theta}=\left.\left(\frac{1}{r} \frac{\partial \Phi_{S}}{\partial \theta}\right)\right|_{r=R_{1}}=-U_{1} \sum_{n=1}^{N}(-1)^{n}\left(2+\frac{1}{n}\right)\left[A_{n}+\frac{1}{2} \delta(n-1)\right] \frac{\partial p_{n 1}^{1}}{\partial \theta} \cos \varphi \\
& v_{\varphi}=\left.\left(\frac{1}{r \sin \theta} \frac{\partial \Phi_{S}}{\partial \varphi}\right)\right|_{r=R_{1}}=U_{1} \sum_{n=1}^{N}(-1)^{n}\left(2+\frac{1}{n}\right)\left[A_{n}+\frac{1}{2} \delta(n-1)\right] p_{n 1}^{1} \frac{\sin \varphi}{\sin \theta}
\end{aligned}
$$

The net forces on the sphere are given as

$$
\begin{aligned}
& F_{x}=\int_{A_{1}} \delta p \mathbf{i} \cdot(-\mathbf{n}) \mathrm{d} A_{1}=-\int_{A_{1}} \delta p \sin \theta \cos \varphi \mathrm{d} A_{1}=0 \\
& F_{y}=\int_{A_{1}} \delta p \mathbf{j} \cdot(-\mathbf{n}) \mathrm{d} A_{1}=-\int_{A_{1}} \delta p \sin \theta \sin \varphi \mathrm{d} A_{1}=0 \\
& F_{z}=\int_{A_{1}} \delta p \mathbf{k} \cdot(-\mathbf{n}) \mathrm{d} A_{1}=-\int_{A_{1}} \delta p \cos \theta \mathrm{d} A_{1} .
\end{aligned}
$$

Substituting (18) into (17), and the result into (21) and performing the integration yields

$$
F_{z}=-2 \pi \rho_{1} R^{2} U_{1}^{2} \sum_{n=1}^{N} n(n+2)^{2}\left[A_{n}+\frac{1}{2} \delta(n-1)\right] A_{n+1}=-\pi \rho_{1} R^{2} U_{1}^{2} f,
$$




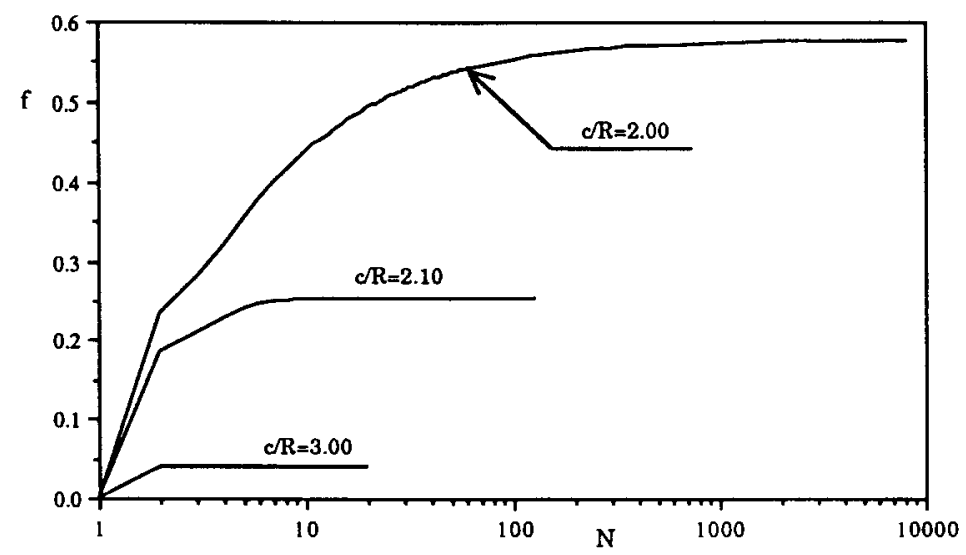

Fig. 5. Lift force coefficient $f$ vs. the number of terms $N$ in velocity potential for two identical spheres with different center distance.

where $f$ is the lift coefficient expressed as

$$
f=2 \sum_{n=1}^{N} n(n+2)^{2}\left[A_{n}+\frac{1}{2} \delta(n-1)\right] A_{n+1} .
$$

The negative sign in (22) indicates that two spheres moving perpendicularly to the line joining their centers attract each other, which is in contrast to the observed and numerically computed results for viscous flow, where the lift is reported to be positive with spheres repulsing each other [1-4]. The lift force coefficient for various truncations are plotted in Fig. 5 for two identical spheres with three different separations.

Figure 5 shows that $f$ increases to a finite limit as $N$ becomes large, and that the number $N$ at which $f$ effectively reaches this limit decreases as the separation increases. Examination of the numerical results reveals that at $N=4, f$ is accurate to the 8 th and 5 th digits for the cases of $c=10 R$ and $c=5 R$, respectively; and at $N=15, f$ is accurate to the 11th and 4th digits for the cases of $c=3 R$ and $c=2.1 R$, respectively; while for the case of two touching spheres $(c=2 R), f$ appears to be accurate only to the second digit at $N=500$, and also even when $N=2000$. It may also be noted from Fig. 5 that, for the case of two touching spheres, the value of $f$ for $N=2000$ is more than eleven per cent (11\%) greater than that for $N=30$ and $.3 \%$ greater than that for $N=1000$.

In order to examine the convergence of $f$ with respect to $N$ for identical touching spheres, $f$ is computed from (23) as a function of $N$, and tabulated in Table 3, along with the differences and the ratio of the differences as $N$ doubles.

Table 3. $f$ computed directly from Eq. (23) for the cases of two identical touching spheres.

\begin{tabular}{rlll}
\hline \multicolumn{1}{r}{$N$} & $f$ & $f(2 N)-f(N)$ & $(f(4 N)-f(2 N)) /(f(2 N)-f(N))$ \\
\hline 512 & 0.571010109557 & & \\
1024 & 0.573927458515 & $0.291734895825 \mathrm{E}-02$ & \\
2048 & 0.575592350152 & $0.166489163645 \mathrm{E}-02$ & 0.570686489780 \\
4096 & 0.576538568692 & $0.946218540156 \mathrm{E}-03$ & 0.568336412678 \\
8192 & 0.577074832251 & $0.536263559049 \mathrm{E}-03$ & 0.566743871834 \\
16384 & 0.577378171456 & $0.303339205584 \mathrm{E}-03$ & 0.565653213733 \\
32768 & 0.577549527750 & $0.171356293326 \mathrm{E}-03$ & 0.564899921183 \\
65536 & 0.577646237175 & $0.967094252200 \mathrm{E}-04$ & 0.564376267384 \\
& & & \\
\hline
\end{tabular}


Table 4. $f$ computed from Table 3, using Richardson's extrapolation.

\begin{tabular}{rlll}
\hline \multicolumn{1}{l}{$N$} & $f$ & $f(2 N)-f(N)$ & $(f(4 N)-f(2 N)) /(f(2 N)-f(N))$ \\
\hline 512 & 0.571010109557 & & \\
1024 & 0.574899908168 & $0.388979861100 \mathrm{E}-02$ & \\
2048 & 0.576230474421 & $0.133056625335 \mathrm{E}-02$ & 0.342065589098 \\
4096 & 0.576911730216 & $0.681255795117 \mathrm{E}-03$ & 0.512004414214 \\
8192 & 0.577287714378 & $0.375984161995 \mathrm{E}-03$ & 0.551898662279 \\
16384 & 0.577498764962 & $0.211050583823 \mathrm{E}-03$ & 0.561328388683 \\
32768 & 0.577617668134 & $0.118903172122 \mathrm{E}-03$ & 0.563387079856 \\
65536 & 0.577684693386 & $0.670252513600 \mathrm{E}-04$ & 0.563696074410 \\
\hline
\end{tabular}

It appears from Table 3 that the convergence of $f$ with respect to $N$ is slow. The values of the computed lift coefficient in Table 3 can be improved somewhat by Richardson's extrapolation [14], taking the errors to be in even orders of $1 / N$. The extrapolated $f \mathrm{~s}$, their differences, and the ratio of the differences as $N$ doubles are given in Table 4. The $f$ values for each $N$ in Table 4 are the extrapolated results using $f$ at $N$ and above $N$ in Table 3, except at $N=512$, which is the same as in Table 3. For example, the value of $f$ for $N=2048$ in Table 4 is the extrapolation result of using values of $f$ at $N=512,1024$ and 2048 in Table 3 .

Comparing the values of $f$ in Tables 3 and 4 , it is seen that at any given $N$ the extrapolation results in some improvement over the computed $f$. But a more important observation is that for any given $N$ the extrapolated value in Table 4 is still smaller than the value at $2 N$ in Table 3 . For example, at $N=8192$ the value in Table 4 is greater than that in Table 3, but still smaller than that at $N=16384$ in Table 3. It can therefore be argued that the extrapolation improves the directly computed $f$, but always underestimates it. By examining the ratio of the increases in Tables 3 and 4 , it is noted that this ratio is decreasing in Table 3 and increasing in Table 4. Assuming that the same trends will continue as $N$ increases in both cases, and using $\Delta f$ and $K$ to denote $f(2 N)-f(N)$ and $(f(4 N)-f(2 N)) /$ $(f(2 N)-f(N))$, respectively at $N=65536$, it is found that

$$
f_{\infty}<f_{65536}+\Delta f \sum_{i=1}^{\infty} K^{i}=0.57777153
$$

from Table 3 , and

$$
f_{\infty}>f_{65536}+\Delta f \sum_{i=1}^{\infty} K^{i}=0.57777129
$$

from Table 4, which form the apparent upper and lower bounds for $f$. Therefore the lift force coefficient for identical touching spheres is 0.577771 .

$f$ as computed from (23) is plotted in Fig. 6 for two identical spheres with varying center distances. It is seen that when the center distances between two identical spheres is 10 times the radius or ( 5 radii from a plane) the interaction force between the two is almost negligible. On the other hand the spheres are essentially touching when they approach within $10^{-5}$ of each other.

\section{Conclusions}

Successive imaging employing spherical harmonics produces an explicit general expression for the velocity potential for two spheres moving perpendicularly to the line joining their 


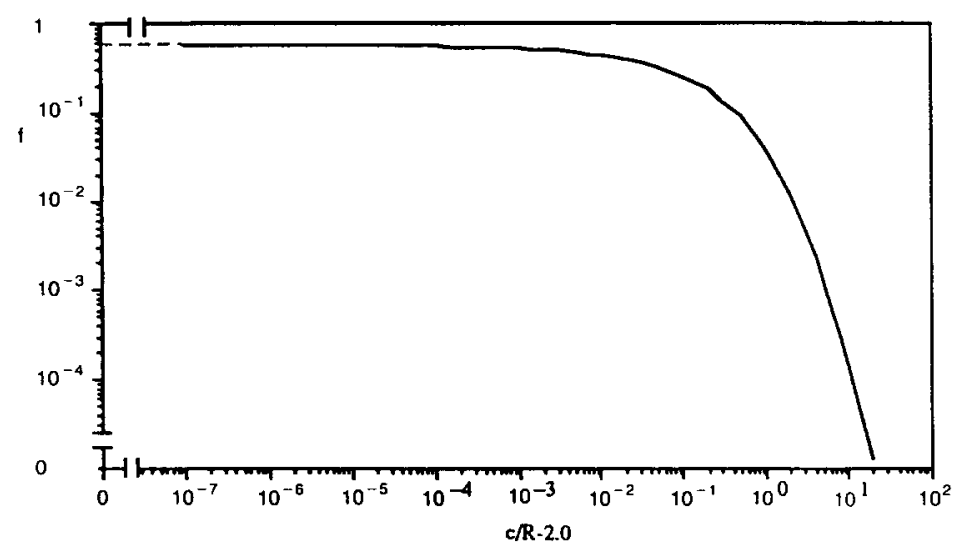

Fig. 6. Lift force coefficient $f$ versus separations.

centers. The appropriateness of the truncation is evaluated by the residual normal velocity at $\varphi=0$, and between $\theta=0$ and $\pi$ on the surface of one of the spheres. The evaluations show that the region near $\theta=\pi$ has the most deviation, and the more terms in the potential the narrower this region and the smaller the deviation. The evaluations also show that if a $N$-term expression for the potential is desired, the truncation should also be equal to $N$. For the evaluation of the interacting force for the case of two identical spheres or one sphere in the vicinity of a solid wall, a four term potential approximation may be satisfactory for $c>5 R$, and the presence of the second sphere or a wall may be neglected when $c>10 R$. However, many more terms along with accelerated computing procedures are required for the potential as $c$ nears $2 R$. We estimate the lift force coefficient for touching spheres to be 0.577771 compared to the most accurate earlier estimate of 0.51435 by Miloh [12].

\section{Appendix}

As stated in section 2 above, the total velocity potential is the sum of the two individual potentials arising from each of the spheres, and can be expressed in the form

$$
\Phi=\left(\Phi_{1}+\Phi_{2}\right) \cos \varphi
$$

Since the two potentials are symbolically symmetrical even when the two spheres are of different sizes and at different velocities, the procedure for constructing the potentials will be given for the sphere centered at $O_{1}$ only.

(3) and (4), from Basset [6], are repeated here for clarity:

$$
\begin{aligned}
& \frac{p_{n 2}^{1}}{r_{2}^{n+1}}=\frac{r_{1}}{(n-1) ! c^{n+2}} \sum_{k=0}^{\infty}(-1)^{k} \frac{(n+1+k) !}{(2+k) !}\left(\frac{r_{1}}{c}\right)^{k} p_{1+k, 1}^{1},\left(r_{1}<c\right), \\
& \frac{(-1)^{n-1} p_{n 1}^{1}}{r_{1}^{n+1}}=\frac{r_{2}}{(n-1) ! c^{n+2}} \sum_{k=0}^{\infty} \frac{(n+1+k) !}{(2+k) !}\left(\frac{r_{2}}{c}\right)^{k} p_{1+k, 2}^{1},\left(r_{2}<c\right),
\end{aligned}
$$

where $p_{n 1}^{1}$ is the first order $n$ th-degree Legendre polynomial with the origin at $O_{1}$ and $p_{n 2}^{1}$ is that with the origin at $O_{2} . \Phi_{1}$ must satisfy the following boundary conditions, from (2): 


$$
\begin{aligned}
& \left.\frac{\partial \Phi_{1}}{\partial r_{1}}\right|_{r_{1}=R_{1}}=-U_{1} p_{11}^{1}, \\
& \left.\frac{\partial \Phi_{1}}{\partial r_{2}}\right|_{r_{2}=R_{2}}=0 .
\end{aligned}
$$

The procedure for constructing $\Phi_{1}$ is begun by approximating it with the well-known potential for a single sphere moving in an infinite quiescent incompressible ideal fluid

$$
\Phi_{1}=U_{1} \frac{R_{1}^{3}}{2 r_{1}^{2}} p_{11}^{1}
$$

which satisfies (A.4). Now (A.3) is used to express (A.6) in terms of the coordinates associated with $\mathrm{O}_{2}$ in the vicinity of the sphere centered at $\mathrm{O}_{2}$ :

$$
\Phi_{1}=U_{1} \frac{D_{1}^{3}}{2} \sum_{i=1}^{\infty} \frac{r_{2}^{i}}{c^{i-1}} p_{i 2}^{1}
$$

where $D_{1}=R_{1} / c$.

It is obvious that (A.7) does not satisfy the boundary condition (A.5), so term (A.8) is added to (A.7):

$$
U_{1} \frac{D_{1}^{3}}{2} \sum_{i=1}^{\infty} \frac{i}{i+1} \frac{R_{2}^{2 i+1}}{r_{2}^{i+1}} \frac{p_{i 2}^{1}}{c^{i-1}}
$$

$\Phi_{1}$ in the vicinity of the sphere $O_{2}$ is now given by

$$
\Phi_{1}=U_{1} \frac{D_{1}^{3}}{2} \sum_{i=1}^{\infty}\left(r_{2}^{i}+\frac{i}{i+1} \frac{R_{2}^{2 i+1}}{r_{2}^{i+1}}\right) \frac{p_{i 2}^{1}}{c^{i-1}} .
$$

Although (A.9) now satisfies the boundary condition (A.5), it will not satisfy (A.4). This is accomplished by using (A.2) to express (A.8) in terms of the coordinates associated with $O_{1}$ in the vicinity of the sphere centered at $O_{1}$, and adding the result to (A.6):

$$
\Phi_{1}=U_{1} \frac{R_{1}^{3}}{2 r_{1}^{2}} p_{11}^{1}+\frac{D_{1}^{3} U_{1}}{2} \sum_{j=1}^{\infty} \sum_{i=1}^{\infty} \frac{(-1)^{j-1} r_{1}^{j}}{(j+1) ! c^{j-1}} p_{j 1}^{1} \frac{i^{2}(i+j) !}{(i+1) !} D_{2}^{2 i+1}
$$

To satisfy (A.4), it is necessary to add (A.11) to (A.10);

$$
\frac{D_{1}^{3} U_{1}}{2} \sum_{j=1}^{\infty} \sum_{i=1}^{\infty} \frac{(-1)^{j-1} j}{(j+1)(j+1) !} \frac{R_{1}^{2 j+1}}{c^{j-1}} \frac{p_{j 1}^{1}}{r_{1}^{j+1}} \frac{i^{2}(i+j) !}{(i+1) !} D_{2}^{2 i+1}
$$

The velocity potential in the vicinity of the sphere $O_{1}$ is now

$$
\Phi_{1}=U_{1} \frac{R_{1}^{3}}{2 r_{1}^{2}} p_{11}^{1}+\frac{D_{1}^{3} U_{1}}{2} \sum_{j=1}^{\infty} \sum_{i=1}^{\infty} \frac{(-1)^{j-1}}{(j+1) !}\left(r_{1}^{j}+\frac{j}{j+1} \frac{R_{1}^{2 j+1}}{r_{1}^{j+1}}\right) \frac{p_{j 1}^{1}}{c^{j-1}} \frac{i^{2}(i+j) !}{(i+1) !} D_{2}^{2 i+1}
$$

which will now satisfy (A.4) but not (A.5). 
It should be noted that since (A.2) and (A.3) are valid only in the vicinities of one of the two spheres, (A.7), (A.9), (A.10) and (A.12) are correspondingly valid only in the vicinity of the sphere stated. The potential represented by (A.12) valid everywhere in the flow field is obtained by summing (A.6), (A.8) and (A.11):

$$
\begin{aligned}
\Phi_{1}= & U_{1} \frac{R_{1}^{3}}{2 r_{1}^{2}} p_{11}^{1}+U_{1} \frac{D_{1}^{3}}{2} \sum_{i=1}^{\infty} \frac{i}{i+1} D_{2}^{i-1} R_{2}^{i+2} \frac{p_{i 2}^{1}}{r_{2}^{i+1}} \\
& +\frac{D_{1}^{3} U_{1}}{2} \sum_{j=1}^{\infty} \sum_{i=1}^{\infty}(-1)^{j-1} \frac{j}{(j+1)(j+1) !} D_{1}^{j-1} R_{1}^{j+2} \frac{p_{j 1}^{1}}{r_{1}^{j+1}} \frac{i^{2}(i+j) !}{(i+1) !} D_{2}^{2 i+1} .
\end{aligned}
$$

The above procedure is repeated by alternately satisfying (A.4) and (A.5), and yields:

$$
\begin{aligned}
\Phi_{1}= & U_{1} \frac{R_{1}^{3}}{2 r_{1}^{2}} p_{11}^{1}+U_{1} \frac{D_{1}^{3}}{2}\left\{\sum_{i=1}^{\infty} \frac{i}{i+1} D_{2}^{i-1} R_{2}^{i+2} \frac{p_{i 2}^{1}}{r_{2}^{i+1}}\right. \\
& +\sum_{j=1}^{\infty} \sum_{i=1}^{\infty}(-1)^{j-1} \frac{j}{(j+1)(j+1) !} D_{1}^{j-1} R_{1}^{j+2} \frac{p_{j 1}^{1}}{r_{1}^{j+1}} \frac{i^{2}(i+j) !}{(i+1) !} D_{2}^{2 i+1} \\
& +\sum_{k=1}^{\infty} \sum_{j=1}^{\infty} \sum_{i=1}^{\infty} \frac{k}{(k+1)(k+1) !} D_{2}^{k-1} R_{2}^{k+2} \frac{p_{k 2}^{1}}{r_{2}^{k+1}} \frac{j^{2}(j+k) !}{((j+1) !)^{2}} D_{1}^{2 j+1} \frac{i^{2}(i+j) !}{(i+1) !} D_{2}^{2 i+1} \\
& +\sum_{l=1}^{\infty} \sum_{k=1}^{\infty} \sum_{j=1}^{\infty} \sum_{i=1}^{\infty}(-1)^{l-1} \frac{1}{(l+1)(l+1) !} D_{1}^{l-1} R_{1}^{l+2} \frac{p_{l 1}^{1} k^{2}(k+l) !}{r_{1}^{l+1}((k+1) !)^{2}} D_{2}^{2 k+1} \frac{j^{2}(j+k) !}{((j+1) !)^{2}} \\
& \left.\cdot D_{1}^{2 j+1} \frac{i^{2}(i+j) !}{(i+1) !} D_{2}^{2 i+1}+\ldots\right\},
\end{aligned}
$$

which is given in a generalized form as (5) in the main text.

\section{Acknowledgements}

This work was supported under NASA Grant NAG3-589. The assistance of Mr John McQuillan and Mr Richard Vernon are gratefully acknowledged.

\section{References}

1. D. Leighton and A. Acrivos, The lift on a small sphere touching a plane in the presence of a simple shear flow. ZAMP 36 (1985) 174-178.

2. D.A. Drew, The lift force on a small sphere in the presence of a wall. J. of Chemical Engineering Science 43 (4) (1988) 769-773.

3. P. Cherukat and J.B. McLaughlin, Wall-induced lift on a sphere. Int. J. Multiphase Flow 16 (5) (1990) $899-907$.

4. P. Vasseur and R.G. Cox, The lateral migration of spherical particles sedimenting in a stagnant bounded fluid. J. Fluid Mech. 80 (1977) 561-591.

5. W.M. Hicks, On the motion of two spheres in a fluid. Philosophical Transactions. Royal Society of London 171 (1880) 455-492.

6. A.B. Basset, On the Motion of Two Spheres in a Liquid, and Allied Problems. Proceedings, Mathematical Society 18 (1887) 369-377.

7. R.A. Herman, On the motion of two spheres in fluid and allied problems. Quartly Journal of Pure and Applied Mathematics xxii (1887) 204-262. 
8. D. Endo, The Force on Two Spheres Placed in Uniform Flow. Proc. Phys.-Math. Soc. Japan 20 (1938) 667-703.

9. J.D. Love, Dielectric sphere-sphere and sphere-plane problems in electrostatics. Quartly Journal of Mech. Appl. Math. 28 (4) (1975) 449-471.

10. R.D. Small and D. Weihs, J. of Applied Mechanics. Trans. A.S.M.E 42 (1975) 763.

11. O.V. Voinov, On the motion of two spheres in a perfect fluid. J. of Applied Mathematics and Mechanics 33 (4) (1969) 638-646.

12. T. Miloh, Hydrodynamics of deformable contiguous spherical shapes in an incompressible inviscid fluid. $J$. of Engineering Mathematics 11 (4) (1977) 349-372.

13. E.W. Hobson, Theory of spherical and ellipsoidal harmonics. Cambridge University Press (1931).

14. R.L. Burden, and J.D. Faires, Numerical Analysis, 3rd edn. Prindle, Weber \& Schmidt, 1985. 